\title{
PRE- AND POST-PERIHELION SPECTROSCOPIC OBSERVATIONS OF COMET KOHOUTEK (1973f)
}

S. Wyckoff and P Wehinger

\begin{abstract}
Twenty pre- and post-perihelion calibrated image-tube spectrograms (3400-9000A; 75 and $150 \mathrm{~A} / \mathrm{mm})$ were obtained of Comet Kohoutek covering a range in heliocentric distances of 0.9 to 0.4 a.u. (30 November $1973-14$ February 1974). Total band intensities have been measured for the $\mathrm{CN}, \mathrm{C}_{2}$ and $\mathrm{NH}_{2}$ bands in the coma spectrum, and for the $\mathrm{CO}^{+}$and $\mathrm{H}_{2} \mathrm{O}^{+}$bands in the tail spectrum. Changes in the total band intensities as a function of heliocentric distance are discussed, as are the pre- and post-perihelion characteristics of the spectra. The line intensity distributions within individual bands of the heteronuclear molecules (with large dipole moments) indicate very low rotational "temperatures" as expected. The measured band intensity ratios of the $A^{2} \pi-X^{2} \Sigma$ (red) $\mathrm{CN}$ bands and the $\mathrm{B}^{2} \Sigma-\mathrm{A}^{2} \pi$ (violet) $\mathrm{CN}$ bands are compared with predicted band intensity ratios calculated by T. Danks and C. A rpigny (1973 Astron. and Astrophys. 29, 347). Finally column densities are determined for $\mathrm{CN}$ and $\mathrm{C}_{2}$ in the coma and $\mathrm{CO}^{+}$in the tail of Comet Kohoutek at various heliocentric distances.
\end{abstract}




\section{DISCUSSION}

W. Jackson: Could you tell us your excitation temperature? How can you define a temperature of a comet when the collision time is long compared to the radiative lifetime of the vibrationally excited $\mathrm{H}_{2} \mathrm{O}^{+}$?

P. Wehinger: Okay, I got your question.

(Laughter.)

It is a standard question.

The point is that this is a kind of artificial parameter in which the - say the Boltzman equation produces or provides a very good approximation for the line and band intensities. And ideally we would like to do this by calculating the population of each level from a large set of equations. And it rapidly becomes an intractable problem.

W. Jackson: I mean, it looked real good, but I don't know whether that was fortuitous or whether there are enough variations to the parameters that you put in that it will fit no matter what you do.

Voice: Couldn't you say that this is an effective radiation temperature?

B. Donn: I don't quite follow something.

In your first paper you said the $\mathrm{H}_{2} \mathrm{O}$ plus is produced by photoionization excitation. And this is what produced the relative intensity that you showed.

Now you are saying that the relative intensities within a band are a temperature effect.

I can't put these two together.

B. Herzberg: Yes, I would also like to comment on that, if I may add to what he said.

It seems to me that if this temperature has any meaning whatever, that is, if there is a radiation temperature, it means that the $\mathrm{H}_{2} \mathrm{O}^{+}$ions radiate their rotational energy and therefore bring the temperature down.

If the excited $\mathrm{H}_{2} \mathrm{O}^{+}$ions were formed by photoionization you wouldn't see this this temperature, but rather the temperature of the original neutral $\mathrm{H}_{2} \mathrm{O}$ molecule. 


\section{DISCUSSION (Continued)}

So I find a little contradiction between those two.

P. Wehinger: Okay.

I appreciate your suggestion.

I should like to ask to explain why we don't see the higher vibronic bands, beyond the 10-0 band.

These should be detectable. They are in a region that is clear of the $\mathrm{C}_{2}$ Swan bands; particularly the 11-0 band is in a region that has no strong, overlapping contaminants. And if it were there - and we have very well-exposed spectra, and we should be able to see it. We just don't.

We don't see any of the other higher bands.

Voice: Does anyone know what the dissociation energy of $\mathrm{H}_{2} \mathrm{O}^{+}$is, because it is just conceivable that they are above the dissociation limit in these particular levels and thus are difficult to observe under low pressure conditions, while in the lab they are observable.

P. Wehinger: Yes.

This may be the case. I could check against the numbers.

Voice: I don't know if anybody knows.

M. K. Wallis: Your slide comparing the observed band intensities with predictions from ionization and fluorescence processes uses the $(9,0)$ band intensity for nomalization. The conclusion favoring ionization would not be so clear if another band were chosen. Is there any good reason for choosing the $(9,0)$ band, such as absence of Greenstein effect?

P. Wehinger: I don't know that there would be. We could have chosen say the 8-0 or 7-0 band, - it wasn't done as any kind of fortuitous argument.

D. J. Malaise: From your computation you conclude that the $\mathrm{H}_{2} \mathrm{O}^{+}$is produced directly by photoionization. Now, Benvenuti has shown us that he has taken spectra in the head and in the tail.

And, of course, in the tail it cannot be produced by photoionization of $\mathrm{H}_{2} \mathrm{O}$ because for that to be, $\mathrm{H}_{2} \mathrm{O}$ should be in the tail, which is not conceivable. 


\section{DISCUSSION (Continued)}

So, is it possible that Benvenuti can tell us whether the ratio of the lines of the bands is different in the head and in the tail?

And secondly, you surely cannot have $\mathrm{H}_{2} \mathrm{O}^{+}$intensity due only to this photoionization.

P. Wehinger: I said that it was primarily photoionization.

D. J. Malaise: Yes, because you observe it in the tail. And in the tail it is surely excited by fluorescence. So it has to be at least partly excited by fluorescence in the head, also.

P. Wehinger: 10 percent.

D. J. Malaise: I don't know.

It should be very interesting to estimate, shouldn't it?

P. Wehinger: Yes.

D. T. Malaise: And then you infer from the intensity of the band and from the process of exciting the bands, the production rate of the ions. But, if you know the cross-section and so on, you should be able also to estimate the density of water, and what the part of the water is photoionized.

Do you have these figures - An estimate of what percentage of the water is ionized to produce these $\mathrm{H}_{2} \mathrm{O}^{+}$bands?

P. Wehinger: I don't have them with me at the moment.

D. J. Malaise: The point is that $\mathrm{H}_{2} \mathrm{O}$ is already dissociated to account for $\mathrm{OH}$ and $\mathrm{H}$, and now if you want to account for $\mathrm{H}_{2} \mathrm{O}^{+}$by ionizing it, we need more water.

C. Cosmovici: I think that the ionization potential of water is higher than the dissociation potential. So, in this case you will have a dissociation rate which is much higher than the photoionization rate.

And do you know the percentage of dissociated molecules compared to the photoionized molecules? 
DISCUSSION (Continued)

P. Wehinger: I can give you a rough figure, for discussion purposes, something like 10 to 1 .

C. Cosmovici: 10 to 1 .

But I think more than 1000 kilometers from the nucleus, all parent molecules would be photodissociated. In any case the ionized water, you see, would be formed outside 1000 kilometers from the nucleus.

So it would be very interesting to know how many molecules would be dissociated in this range, and how many would be photoionized. But I don't know, maybe this is not a process for ionization. Maybe collisional ionization- I don't know that.

But I think this question should be discussed a little bit.

W. Jackson: In the first place, most of the water has to be dissociated in the first continuum. It can't be 10 percent.

The solar flux -

P. Wehinger: No, what I was saying is that if you have some amount of water- it is 10 to $1-10$ times as much is dissociated as is photoionized.

W. Jackson: That 90 percent, even that is overestimated by maybe one or two orders of magnitude. I think Delsemme can give you the exact number.

A. H. Delsemme: Well I looked crudely into that problem sometime ago already, and 99 plus percent of the water is dissociated and less than 1 percent is ionized.

I have more exact figures, but roughly that is the crude answer - taking into account the wavelength distribution of the solar continuum.

Voice: There is a basic discrepancy with another estimate of the solar production rate of $\mathrm{H}_{2} \mathrm{O}^{+}$, which is to take the rocket measurements of the $\mathrm{H}$ production rate, which we assume is produced by dissociation. You get the same number as saying that the hydrogen production is twice the $\mathrm{H}_{2} \mathrm{O}$ production rate. We find that according to his numbers you need all of the $\mathrm{H}_{2} \mathrm{O}$ going into $\mathrm{H}_{2} \mathrm{O}^{+}$.

So I think there is a major discrepancy in the data being presented and the cause of that is probably in the calculation of these source or $q$ factors for computing the rate at which the photoionization takes place. 


\section{DISCUSSION (Continued)}

W. Jackson: When you estimate the total production of $\mathrm{H}_{2} \mathrm{O}^{+}$, you have to have an oscillator strength. You can't use the relative line strength measurements measured in the lab, to get an estimate of the total amount of $\mathrm{H}_{2} \mathrm{O}^{+}$.

You have to know what the transition probability is from the excited state to the ground state.

And that number is not known as far as -

G. Herzberg: For $\mathrm{H}_{2} \mathrm{O}$ ? - Yes it is.

W. Jackson: Yes? It has been measured?

G. Herzberg: The lifetime is known. I believe it is 800 nanoseconds.

W. Jackson: You used that number?

P. Wehinger: And you should also point out that there is a branching ratio that has to be accounted for because of the three lower levels.

And we are only looking at one of the lower levels.

W. Jackson: And when you do all of that you still get as much $\mathrm{H}_{2} \mathrm{O}^{+}-$ you would require enough water, such that most of the water undergoes photoionization, and that is in direct contradiction with the Lyman alpha measurement in the ultraviolet region.

H. Keller: This table we saw, this is $\mathrm{H}_{2} \mathrm{O}^{+}$or $\mathrm{H}_{2} \mathrm{O}$ production rate?

P. Wehinger: $\mathrm{H}_{2} \mathrm{O}^{+}$.

I'm sorry, it is $\mathrm{H}_{2} \mathrm{O}$.

Voice: Oh, okay. Then there is no contradiction.

M. Dubin: Talking about tables, in one of your tables you gave an equivalent of radius of the nucleus.

And you showed in the case of comet Kohoutek, a difference in the radius of the nucleus that was substantial. You show something like 7 kilometers down to a few kilometers, one or two kilometers, after perihelion.

Now that kind of a model won't fit. That kind of a transition in radius doesn't fit, say, the Whipple model. 


\section{DISCUSSION (Continued)}

How can you adjust that radius to keep it only a very small change, in line with your observation, where the radius is essentially constant? of two.

Voice: 7.6 kilometers versus 2 kilometers.

M. Dubin: That is still too much. 